\title{
Fast Detection of Ventricular Fibrillation and Ventricular Tachycardia in 1-Lead ECG from Three-Second Blocks
}

\author{
Filip Plesinger, Petr Andrla, Ivo Viscor, Josef Halamek and Pavel Jurak \\ Institute of Scientific Instruments of the CAS, Brno, Czech Republic
}

\begin{abstract}
Background: Ventricular tachycardia (VT) is dangerous irregularity of heart rhythm. VT may evolve into ventricular fibrillation (VF) which often leads to cardiac death. Therefore, fast automated detection of $V F / V T$ events is of the utmost importance. Here, we present a method detecting VT and ventricular fibrillation (VF) events suitable for real-time application on continuously incoming ECG data.

Method: We designed a method for detection of VF/VT events in short-time (3 s), 1-lead ECG blocks. Five features are extracted from this block using analysis of ECG spectra, derivatives, amplitude measures and autocorrelation. The extracted features are fed into a logistic regression model showing the probability of a VF/VT event. The model was trained on the public PhysioNet CUDB dataset consisting of 393 automatically selected blocks.

Results: The model (AUC 0.99) showed a sensitivity and specificity of $95 \%$ and $97 \%$, respectively (5-fold cross-validation). The model was tested on the public PhysioNet VFDB dataset, showing specificity and sensitivity of $95 \%$ and $83 \%$, respectively. Both the feature extraction code (Matlab format) and the model are publicly accessible and easy implementation of the logistic regression model predetermines it for real-time applications.
\end{abstract}

\section{Introduction}

Ventricular tachycardia (VT) is life-threatening arrhythmia characterized as several (at least 3-5) ventricular beats at $100 \mathrm{bpm}$ or more. Eventually, it may become ventricular fibrillation (VF), described as chaotic rapid contractions of ventricles. During VF, arterial blood pressure rapidly decreases meaning that there is no blood circulation.

A complication in automated VF and, partially, VT detection is that QRS waveforms might not be detectable by QRS detectors due to their unusual morphology. However, the VF/VT repeating pattern is relatively easy to recognize by specialists. Therefore, existing studies usually focus on using autocorrelation [1], [2], Fast Fourier Transform (FFT) [3], [4] in short windows, digital filtering [5], a Threshold Crossing Intervals algorithm [6], and others. The extracted feature(s) are then checked to pass a threshold [7] or fed into some machine learning approach as shown later on multiple methods (neural networks, bagged trees, supported vector machines, etc.) presented in the PhysioNet Challenge 2015 [8].

In this paper, we present a method for detecting VF/VT events from short time blocks which should be suitable for easy implementation in hand-held devices.

\section{Method}

The presented method is designed to process shorttime (3-sec) blocks of 1-lead ECG files. The ECG signal is detrended, filtered (FFT band pass 1-35 $\mathrm{Hz}$ ) and normalized to the range $<0 ; 1>\left(E C G_{\mathrm{PR}}\right)$. In order to detect a VF/VT event, five features are extracted from this shorttime ECG.

\subsection{Feature extraction}

The first feature - AmpRatio - is based on frequency analysis. We noticed that higher harmonics in VF/VT areas decrease faster in comparison to non-VF/VT areas. Maximal peak is found in FFT spectra (above $2 \mathrm{~Hz}$ ); then an amplitude if its $2^{\text {nd }}$ harmonics is compared to an amplitude of that maximal peak.

The second feature - RatioDiff - compares amplitudes in the filtered signal and its derivative, because a VF/VT signal should contain mostly "steep" areas, while regular ECG does not. RatioDiff is computed as:

$$
\text { RatioDiff }=\frac{P_{90}\left|\operatorname{diff}\left(E C G_{P R}\right)\right|}{P_{90}\left(E C G_{P R}\right)-P_{10}\left(E C G_{P R}\right)}
$$

where $\mathrm{P}$ denotes the $\mathrm{n}^{\text {th }}$ percentile.

The third feature - VR - is computed as the minimal variation range (max-min) of ECG $_{\mathrm{PR}}$ in a $0.15 \mathrm{sec}$ floating window with a 0.075 sec step. 
The fourth feature - Corr85 - is sensitive to the expected cyclic behaviour of VF/VT. The inner third of $E_{P R}$ is correlated through the whole ECG $\mathrm{ER}_{\mathrm{PR}}$ the Corr85 is then computed as the $85^{\text {th }}$ percentile of the correlation result.

The last, fifth feature - MeanPC - is also based on the correlation result used for Corr85. Peaks are extracted from the correlation result (min. distance $0.1 \mathrm{~s}$, min. height Corr85). The MeanPC is then computed as the mean peak amplitude.

\subsection{Model training}

The presented features were extracted for 393 automatically selected blocks from the public CUDB [9] database available at PhysioNet [10]. 194 blocks were labelled as VF, 5 as VT, 184 as normal and 10 as noisy; the original labelling was used. VT and VF blocks formed the positive group, other blocks formed the negative group. If multiple labels belonged to a 3-sec ECG block, then VF/VT labels were preferred. Next, the logistic regression model (5-fold cross-validation) was trained using the Matlab ${ }^{\circledR}$ software version 2017b and Machine Learning and Statistics Toolbox. The generated model is shown in Table 1.

Table 1. Model features. The P-value of features \#1-5 is < 0.001 .

\begin{tabular}{lll}
\hline Number & Text & Estimate \\
\hline & (intercept) & -1.2677 \\
$\# 1$ & AmpRatio & -147.1494 \\
$\# 2$ & RatioDiff & -60.2266 \\
$\# 3$ & VR & 16.2199 \\
$\# 4$ & Corr85 & 12.4347 \\
$\# 5$ & MeanPC & -12.7446 \\
\hline
\end{tabular}

\section{Results}

Training results for the CUDB dataset (5-fold crossvalidation) showed a sensitivity and specificity of 0.95 and 0.97 , respectively (Tab. 2). The presented model was also tested on an independent VFDB dataset [10], showing sensitivity and specificity of 0.95 and 0.83 . Furthermore, Table 2 and Figure 1 present detailed results for specific classes using the VFDB database. Both the VFDB and CUDB databases were sampled at $250 \mathrm{~Hz}$ with 12-bit resolution.

Table 2. Model results on the training (CUDB) and testing (VFDB) datasets.

\begin{tabular}{lll}
\hline Dataset & Sensitivity & Specificity \\
\hline CUDB & 0.95 & 0.97 \\
VFDB & 0.95 & 0.83 \\
\hline
\end{tabular}

Table 3. Test results on the VFDB dataset for specific classes. AF - atrial fibrillation, AS - asystole, BI - PVC bigeminy, $\mathrm{N}$ - normal sinus rhythm, $\mathrm{X}$ - noisy, VF ventricular fibrillation, VT - ventricular tachycardia. SD - standard deviation, CI - confidence interval of mean. * - these CI values were trimmed to the range $<0-1>$.

\begin{tabular}{lllll}
\hline Class & Count & Mean & SD & $95 \%$ CI \\
\hline AF & 7 & 0.001 & 0.003 & $0.000^{*}: 0.003$ \\
AS & 24 & 0.223 & 0.370 & $0.067: 0.379$ \\
BI & 15 & 0.151 & 0.255 & $0.010: 0.292$ \\
N & 37 & 0.234 & 0.347 & $0.117: 0.352$ \\
X & 35 & 0.262 & 0.306 & $0.157: 0.367$ \\
VF & 41 & 0.944 & 0.182 & $0.887: 1.000^{*}$ \\
VT & 82 & 0.903 & 0.218 & $0.855: 0.951$ \\
\hline
\end{tabular}

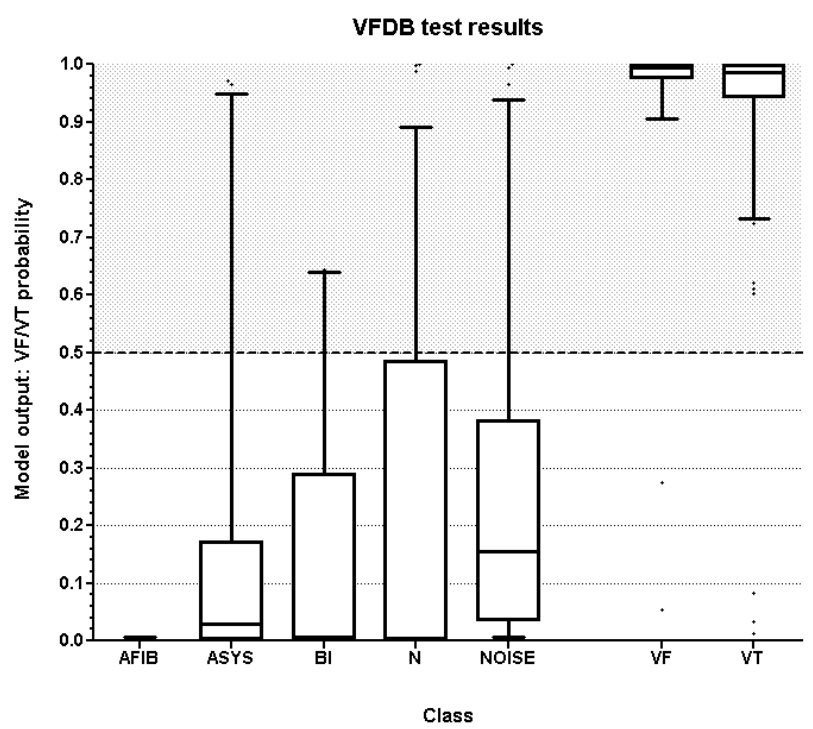

Figure 1. Test set results computed for the VFDB database using the presented logistic regression model. Probabilities over 0.5 were considered VF/VT. AFIB atrial fibrillation, ASYS - asystole, BI - PVC bigeminy, $\mathrm{N}$ - normal sinus rhythm, NOISE - noisy signal, VF ventricular fibrillation, VT - ventricular tachycardia.

Figure 2 shows 12 examples of different ECG blocks processed by the presented method. It also shows feature values as well as method output: probability in a range 0-1 where values higher than 0.5 mean VF/VT classification. 


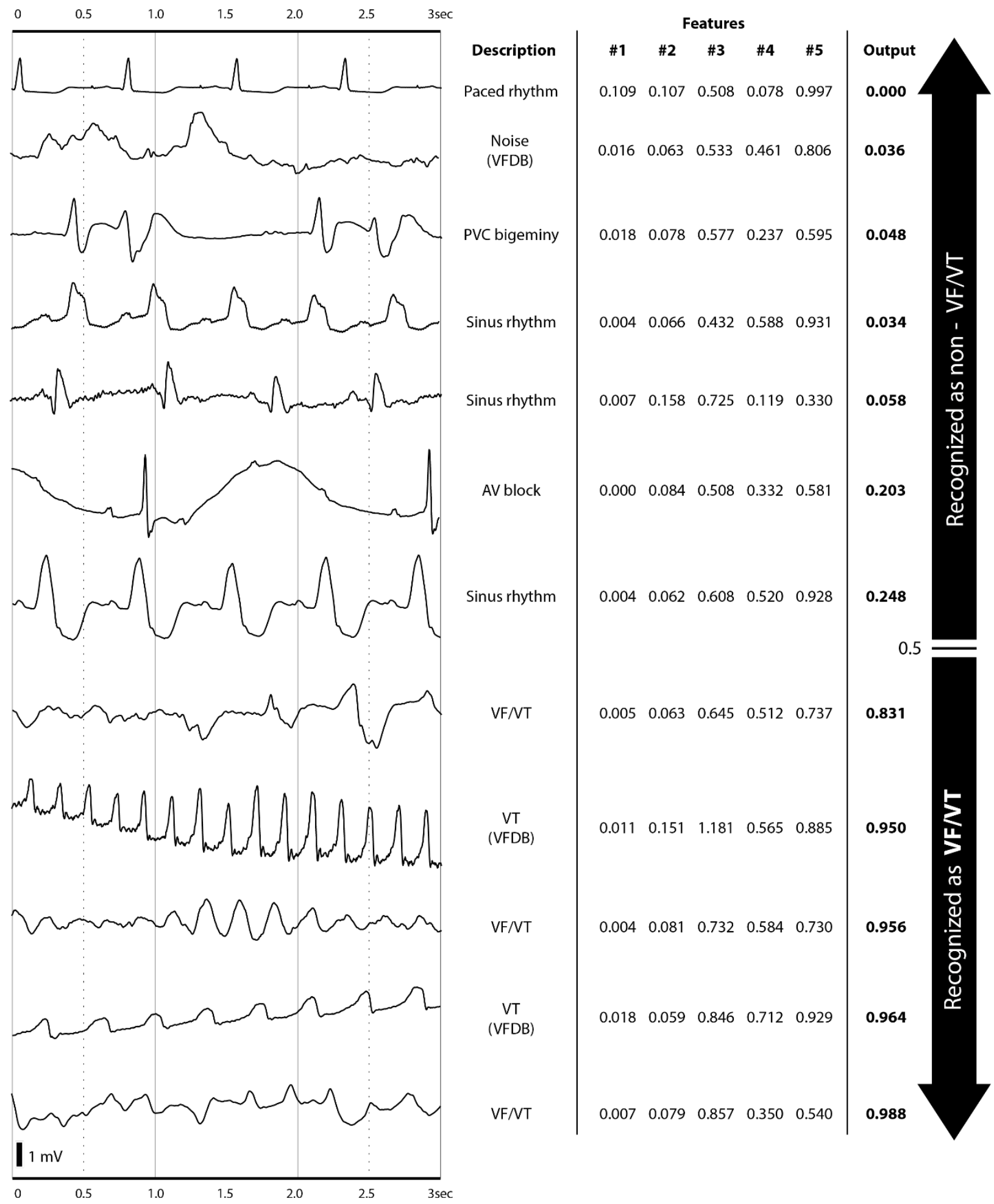

Figure 2: Examples of ECG blocks from training set (generally CUDB database; VFDB database where noted) with extracted feature values and output of logistic regression model. An output value higher than 0.5 refers to VF/VT, otherwise it is considered non-VF/VT. Features are numbered in accordance with Table 1 . The raw signal extracted from CUDB/VFDB databases is shown. 


\section{Discussion}

The presented model performed perfectly during crossvalidation tests on the CUDB dataset. However, although it achieved the same sensitivity on the (testing) VFDB dataset, the results on VFDB showed weaker specificity. A detailed view on specificity showed that the biggest deficit comes from misclassification of normal blocks (spec. $78 \%$ ) and asystolic blocks (79\%). While the lower specificity in normal recordings should be further investigated, in the case of asystoles it may be explained as a side effect of signal normalization. On the other hand, the highest specificity was measured in atrial fibrillation blocks (100\%, though limited by the low number of cases) and noisy blocks (86 \%).

From the point of view of implementation, the logistic regression model is easy to implement. Moreover, processing of 3-sec data blocks is computationally undemanding (if common ECG sampling frequencies are used). Therefore, the presented study may be considered a solution for miniature devices needing quick and automated notification of a VF/VT event.

However, several limitations should be considered. Firstly, both the CUDB and VFDB datasets consisted of a low number of recordings (35 in CUDB, 22 in VFDB). This limits the number of morphological variants of specific arrhythmia events. Also, blocks extracted from the CUDB dataset contain only 5 cases of VT. This hardly affected the test results on VFDB due to the fact that VTs in VFDB usually have a similar morphology to VFs. However, we feel that this similarity between VF and VT morphology in VFDB is the most dangerous limitation of this study. Therefore, we expect decreased sensitivity in ECG recordings when VT is not followed by VF.

Future work should include a parallel model retrained for these VT cases, as we have already found that features \#1 (Tab. 1 - AmpRatio) and \#3 (Tab. 1 - VR) have different trends for VTs acquired from a private dataset (ECG Holters, non-hospitalized patients).

\section{Conclusion}

We presented a logistic regression model detecting $\mathrm{VF} / \mathrm{VT}$ events. The results have shown that it has a strong capability to capture these events from short (3-s) ECG blocks. The low number of features (5) and easy implementation of the logistic regression model are aimed at hand-held devices. The feature extraction code as well as the model are publicly accessible under an MIT licence at Github repository (https://github.com/fplesinger).

\section{Acknowledgements}

This research was supported by project MSM 100651602 by the Czech Academy of Sciences and by project LO1212 by MEYS CR.

\section{References}

[1] S. Kuo and R. Dillman, "Computer Detection of Ventricular Fibrillation," in Computers in Cardiology, 1978, pp. 347-349.

[2] S. Chen, N. V. Thakor, and M. M. Mower, "Ventricular Fibrillation Detection by a Regression Test on the Autocorrelation Function,” Med. Biol. Eng. Comput., vol. 25, no. 3, pp. 241-249, 1987.

[3] Y. Li, J. Bisera, W. Tang, and M. H. Weil, “Automated Detection of Ventricular Fibrillation to Guide Cardiopulmonary Resuscitation," Crit. Pathw. Cardiol., vol. 6, no. 3, pp. 131-134, 2007.

[4] T. Eftestøl, L. Wik, K. Sunde, and P. A. Steen, "Effects of Cardiopulmonary Resuscitation on Predictors of Ventricular Fibrillation Defibrillation Success During Out-of-Hospital Cardiac Arrest,” Circulation, vol. 110, no. 1, pp. 10-15, 2004.

[5] I. Jekova and V. Krasteva, "Real Time Detection of Ventricular Fibrillation and Tachycardia," Physiol. Meas., vol. 25, no. 5, p. 1167, 2004.

[6] N. V. Thakor, Y. S. Zhu, and K. Y. Pan, "Ventricular Tachycardia and Fibrillation Detection by a Sequential Hypothesis Testing Algorithm,” IEEE Trans. Biomed. Eng., vol. 37, no. 9, pp. 837-843, 1990.

[7] F. Plesinger et al., "Taming of the Monitors: Reducing False Alarms in Intensive Care Units,” Physiol. Meas., vol. 37, no. 8, 2016.

[8] G. D. Clifford et al., "The PhysioNet / Computing in Cardiology Challenge 2015: Reducing False Arrhythmia Alarms in the ICU," Comput. Cardiol., vol. 42, pp. 273-276, 2015.

[9] F. M. Nolle et al., "CREI-GARD, a New Concept in Computerized Arrhythmia Monitoring Systems," Comput. Cardiol., pp. 515-518, 1987.

[10] A. L. Goldberger et al., "PhysioBank, PhysioToolkit, and PhysioNet: Components of a New Research Resource for Complex Physiologic Signals.," Circulation, vol. 101, no. 23, pp. E215-E220, 2000.

Address for correspondence:

Filip Plesinger

Institute of Scientific Instruments of the CAS, v.v.i.

Kralovopolska 147,

Brno 612 64, Czech Republic

E-mail address: fplesinger@isibrno.cz 